Säuredissoziation $\left[\mathrm{HNi}_{4}(\mathrm{CO})_{9}\right]^{\ominus} \rightleftarrows\left[\mathrm{Ni}_{4}(\mathrm{CO})_{9}\right]^{2 \ominus}+\mathrm{H}^{\oplus}$ erhält man für die Dissoziationskonstante $K_{s}$ der Säure $\left[\mathrm{HNi}_{4}(\mathrm{CO})_{9}\right]^{\ominus}$ :

$$
\begin{aligned}
K_{\mathrm{s}}=\frac{a_{\mathrm{H}}^{2 \oplus} \cdot C \cdot \gamma}{K_{\mathrm{w}}} & a_{\mathrm{H}}^{\oplus}=\text { Aktivität von } \mathrm{H}^{\oplus} \text { (gemessen), } \\
K_{\mathrm{W}} & =\text { Ionenprodukt des Wassers. }
\end{aligned}
$$$$
K_{\mathrm{s}}=1,12 \cdot 10^{-10} \sim 10^{-10} ; \quad p K_{\mathrm{s}}=10 \text {. }
$$

Der Deutschen Forschungsgemeinschaft und dem Fonds der Chemischen Industrie danken wir für die Unterstützung unserer Arbeiten. Ebenso danken wir der Direktion der B a dis chen Anilin-und Soda-Fabrik A.G., Ludwigs hafen/Rhein, für die Überlassung wertvollen Ausgangsmaterials.

\title{
Über die IR-Spektren ungesättigter N-heterocyclischer Verbindungen
}

\author{
II. Mitt.: Über die CH-Valenzschwingungen monomethylsubstituierter $N$-Heterocyclen* \\ Von A. Roders und H.-H. Perkampus
Aus dem Institut für Physikalische Chemie und Elektrochemie der Technischen Hochschule Hannover
(Z. Naturforschg. 18 b, 600-604 [1963] ; eingegangen am 9. Mai 1963)

\begin{abstract}
Die Lage der Banden von $v \cdot \mathrm{CH}_{\mathrm{al}}$ und $v-\mathrm{CH}_{\mathrm{ar}}$ und ihre maximalen Extinktionskoeffizienten von vierzehn monomethylsubstituierten $N$-Heterocyclen wurde bestimmt. Ein Zusammenhang zwischen der Lage der $v \cdot \mathrm{CH}_{\mathrm{al}}$ und der Kondensationsfähigkeit der Methylgruppe besteht nicht, jedoch deutet sich ein Zusammenhang mit der Intensität an.
\end{abstract}

Die Absorptionsspektren von vierzehn monomethylsubstituierten $N$-Heterocyclen werden im Bereich $2800-3200 \mathrm{~cm}^{-1}$ mit einem hochauflösenden Gitterspektralphotometer aufgenommen und die Lage der Banden der aliphatischen und aromatischen CH-Valenzschwingung bestimmt. Es wurde versucht, eine Korrelation zwischen der Reaktionsfähigkeit der Methylgruppe und der Lage der Banden der aliphatischen CH-Valenzschwingung herzuleiten.

Bei der Bestimmung der Bandenlage der $v-\mathrm{CH}_{\mathrm{a} 1}$ ** von Methylgruppen in polycyclischen aromatischen Kohlenwasserstoffen fanden Badger und Moritz ${ }^{1}$ einen Zusammenhang zwischen der Bandenlage und der freien Valenzzahl (free valence number) ${ }^{2}$ des Ringkohlenstoffatoms, das eine Methylgruppe als Substituenten trägt. Es lag nahe, die Untersuchungen auf methylsubstituierte $N$-Heterocyclen auszudehnen. Vorerst wurden vierzehn Verbindungen vermessen und zwar: 2-, 3-, 4-Methylpyridin, Methylpyrazin, 2-, 4-, 6-, 7-, 8-Methylchinolin, 1-, 3-Methylisochinolin, 2-, 5-, 6-Methylchinoxalin. Die IRSpektren dieser Verbindungen sind bekannt ${ }^{3-8}$, je-

* I. Mitt.: Z. Naturforschg. 15 b, 1 [1960]

** $v-\mathrm{CH}_{\mathrm{al}} \triangleq$ aliphatische CH-Valenzschwingung; $v \cdot \mathrm{CH}_{\mathrm{ar}} \triangleq$ aromatische $\mathrm{CH}$-Valenzschwingung.

1 G. M. Badger u. A. G. Moritz, Spectrochim. Acta [London] 15, 675 [1959].

2 s. z. B. B. A. Streitwieser JR., Molecular Orbital Theory for Organic Chemists, New York, London 1961, S. 329.

3 A. R. Katritzky u. A. R. Hands, J. chem. Soc. [London] $1958,2202$. doch sind eingehende Untersuchungen des Bereichs der $v-\mathrm{CH}_{\mathrm{al}}$ im Hinblick auf eine Korrelation zwischen der Reaktivität der Methylgruppe und der Lage der Banden der $v$ - $\mathrm{CH}_{\mathrm{al}}$ nicht vorgenommen worden.

Die freie Valenzzahl ist nach der MO-Theorie eine der Größen, die eine Abschätzung der Reaktivität der verschiedenen Positionen z. B. in einem aromatischen Kohlenwasserstoff oder einem Heterocyclus erlaubt. Die freien Valenzzahlen der Stammkörper, der hier untersuchten methylsubstituierten $N$-Heterocyclen, sind z. T. bekannt. Da aber genügend experimentelles Material über die Reaktivität der Methylgruppen vorliegt, soll im folgenden die freie Valenzzahl nicht zur Charakterisierung der Reaktivität herangezogen werden. Als Kriterium für die Reaktivität dient die Fähigkeit, mit aromatischen Aldehyden je nach Reaktionsbedingung zu Carbinolen bzw. Styrylverbindungen zu kondensieren.

Es ist bekannt, daß Methylgruppen in $N$-Heterocyclen in $\alpha$ - und $\gamma$-Stellung zum Stickstoff kondensationsfähig sind; sie sind nicht reaktionsfähig in

${ }^{4}$ D. A. Losg u. a., Trans. Faraday Soc. 53, 1171 [1957].

5 A. R. Katritzky u. R. A. Jones, J. chem. Soc. [London] 1960, 2942.

${ }_{6}^{6}$ D. P. Biddiscombe u. a., J. chem. Soc. [London] 1954, 1957.

7 E. Baumgarten, Dissertation, Hannover 1962.

8 H.-H. Perkampus u. A. Roders, Z. Naturforschg. 15 b, 1 [1960], I. Mitt. und A. Roders, Diplomarbeit, Hannover 1959. 
$\beta$-Stellung. Methylgruppen in homocyclischen aromatischen Kohlenwasserstoffen sind ebenfalls nicht kondensationsfähig ${ }^{9}$. Von den hier untersuchten Verbindungen haben eine reaktive Methylgruppe 2-, 4-Methylpyridin, Methylpyrazin, 2-, 4-Methylchinolin, 1-, 3-Methylisochinolin, 2-Methylchinoxalin; eine nichtreaktive Methylgruppe haben 3-Methylpyridin, 6-, 7-, 8-Methylchinolin, 5- und 6-Methylchinoxalin. Die vorgenommene Einteilung kann im allgemeinen als gesichert gelten, so kondensiert 2-Methylchinoxalin mit aromatischen Aldehyden, nicht aber 5- und 6-Methylchinoxalin ${ }^{10}$; jedoch liegen in zwei Fällen sich widersprechende Befunde vor: nach Phillips ${ }^{11}$ kondensiert das Methojodid des 3-Methylpyridins nicht mit $p$-Dimethylaminobenzaldehyd, während CRIPPA und VERDI ${ }^{12}$ eine Kondensation beobachtet haben. Ebenso ist nach Mills und Sмгтн ${ }^{13}$ 3-Methylisochinolin nicht reaktionsfähig; neuere Untersuchungen von ERLENMEYER ${ }^{14}$ beweisen die Reaktionsfähigkeit.

Es soll im folgenden untersucht werden, ob diese Unterschiede in der Kondensationsfähigkeit sich auf die Lage der Banden der $v-\mathrm{CH}_{\mathrm{al}}$ auswirkt. Der Vollständigkeit halber werden auch die Bandenlagen der $v$ - $\mathrm{CH}_{\mathrm{ar}}$ angegeben.

\section{Experimentelles}

Die Methylpyridine, Methylchinoline, Methylisochinoline, das Methylpyrazin und 2-Methylchinoxalin sind im Handel erhältlich. Sie wurden durch Destillation bzw. Umkristallisation gereinigt. 5-Methylchinoxalin wurde aus 2.3-Diaminotoluol und Glyoxalnatriumbisulfit dargestellt ${ }^{15}$. Die Darstellung des 6-Methylchinoxalins ist früher beschrieben worden ${ }^{8}$. Der als Lösungsmittel verwendete Tetrachlorkohlenstoff wurde durch zweimalige fraktionierte Destillation weitgehend von Verunreinigungen befreit, die im Bereich der $v$ - $\mathrm{CH}$ absorbieren. Unter Verwendung von 0,10-cm-Küvetten aus Infrasil wurden $0,12-0,24$ molare Lösungen in Tetrachlorkohlenstoff vermessen. Die Spektren wurden mit dem Unicam SP 700 Spektralphotometer unter Benutzung des Gitters aufgenommen. Die untere Grenze der Registriermöglichkeit liegt bei $2800 \mathrm{~cm}^{-1}$. Die Temperatur betrug $22{ }^{\circ} \mathrm{C}$. Zur Wellenlängeneichung des Meßbereichs wurde unmittelbar vor jeder

9 E. H. Rodp, Chemistry of Carbon Compounds, Amsterdam, London, New York, Princeton 1957, Bd. 4, Teil A.

10 G. M. Bennett u. G. H. Willis, J. chem. Soc. [London] 1928, 1960.

11 A. P. Phillips, J. Amer. chem. Soc. 74, 3296 [1952].

12 G. B. Crippa u. T. Verdi, Ann. chim. applicata 26, 418 [1936].
Messung das Spektrum des Polystyrols aufgenommen. Bei scharfen Banden ist die Lage des Maximums auf $\pm 2 \mathrm{~cm}^{-1}$ genau angebbar.

\section{Ergebnisse}

Das vermessene Intervall von $2800-3200 \mathrm{~cm}^{-1}$ läßt sich in den Erwartungsbereich der $v-\mathrm{CH}_{\mathrm{al}}$ von ca. $2800-3000 \mathrm{~cm}^{-1}$ und den der $v-\mathrm{CH}_{\mathrm{ar}}$ von ca. $3000-3200 \mathrm{~cm}^{-1}$ einteilen ${ }^{16}$. Insbesondere sind Banden bei $2872 \pm 10 \mathrm{~cm}^{-1}$ und $2962 \pm 10 \mathrm{~cm}^{-1}$ charakteristisch für die $\nu$-CH der Methylgruppe ${ }^{17}$.

Bei den untersuchten Verbindungen beobachtet man, wie aus den Abb. $1-14$ hervorgeht, im Bereich der $v-\mathrm{CH}_{\mathrm{al}}$ eine Gruppe von drei Einzelbanden und zwar bei $2987 \pm 10 \mathrm{~cm}^{-1}, 2956 \pm 10 \mathrm{~cm}^{-1}$ und $2924 \pm 6 \mathrm{~cm}^{-1}$; letzterer Bande ist eine schwache Vorbande bzw. Schulter bei $2865 \pm 15 \mathrm{~cm}^{-1}$ vorgelagert. (Die Wellenzahlangaben bezeichnen das Intervall, in dem alle Verbindungen absorbieren. Die Werte sind das arithmetische Mittel aus der jeweils größten und kleinsten Wellenzahl, bei der eine deutlich erkennbare Bande bzw. Schulter auftritt.) Eine zusätzliche Absorption in Form einer schwach ausgeprägten Schulter um $2900 \mathrm{~cm}^{-1}$ zeigen die Verbindungen 4-Methylpyridin, 5-Methylchinoxalin, 4-, 8-Methylchinolin und 3-Methylisochinolin, während die Bande $2987 \pm 10 \mathrm{~cm}^{-1}$ beim 2-Methylpyridin, Methylpyrazin, 2-Methylchinolin, 1-Methylisochinolin und 5-Methylchinoxalin nicht beobachtet wird. Es ist in diesem Zusammenhang interessant, daß die Schulter bei $2900 \mathrm{~cm}^{-1}$ außerdem beim 1-Methylnaphthalin auftritt und daß fast alle Methylderivate, die diese Bande zeigen, bezüglich der Stellung der Methylgruppe am Ringsystem dem 1-Methylnaphthalin ähneln. Die Lage der Absorptionsmaxima der $v$ - $\mathrm{CH}_{\mathrm{al}}$ ist in Tab. 1 zusammengestellt.

Im Bereich der $v-\mathrm{CH}_{\mathrm{ar}}$ beobachtet man eine Gruppe von vier Einzelbanden und zwar: bei $3082 \pm 12 \mathrm{~cm}^{-1}, \quad 3062 \pm 12 \mathrm{~cm}^{-1}, \quad 3034 \pm 8 \mathrm{~cm}^{-1}$ und $3013 \pm 7 \mathrm{~cm}^{-1}$, jedoch gibt es mehrere Verbindungen, die in einem oder zwei dieser Bereiche nicht

13 W. H. Mills u. J. L. B. Smith, J. chem. Soc. [London] 121, 2724.

14 H. Erlenmeyer u. a., Helv. chim. Acta 31, 1978 [1948].

15 J. K. Lundquist, J. chem. Soc. [London] 1953, 2816.

16 E. D. Sснмid, Z. Elektrochem., Ber. Bunsenges. physik. Chem. 66, 53 [1962].

17 L. J. Bellamy, Ultrarotspektrum und chemische Konstitution, Darmstadt 1955. 
Abb. 1.

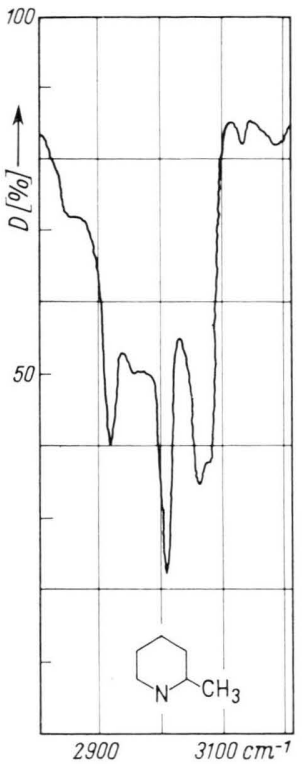

Abb. 2.

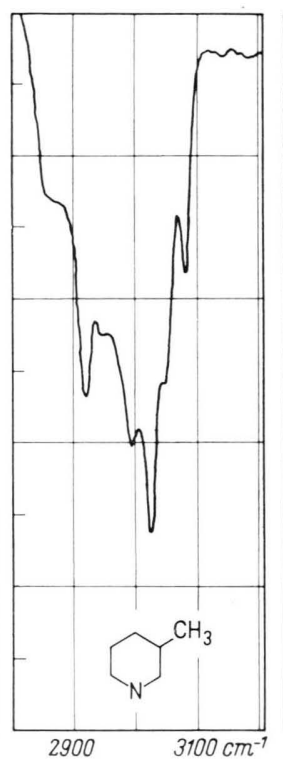

Abb. 3.

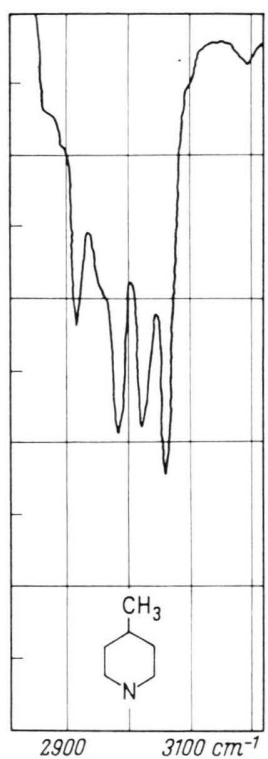

Abb. 4 .

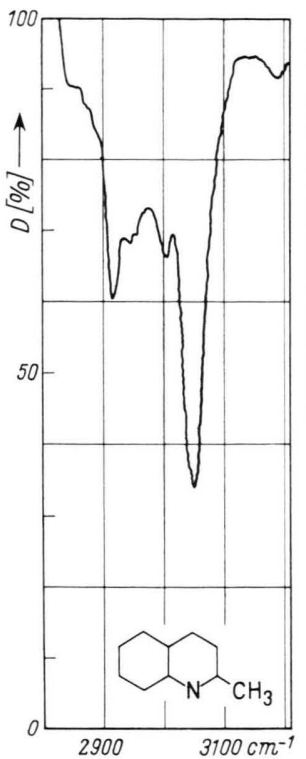

Abb. 5.

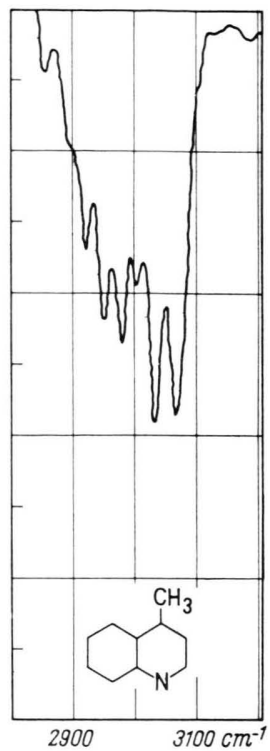

Abb. 1. Banden der CH-Valenzschwingungen von 2-Methylpyridin, $c=0,24$-molar; in Tetrachlorkohlenstoff, $T=22^{\circ} \mathrm{C}$, $d=0,10 \mathrm{~cm}$ (gilt für alle Abbildungen).

Abb. 2. Banden der CH-Valenzschwingungen von 3-Methylpyridin, $c=0,18$-molar.
Abb. 6.

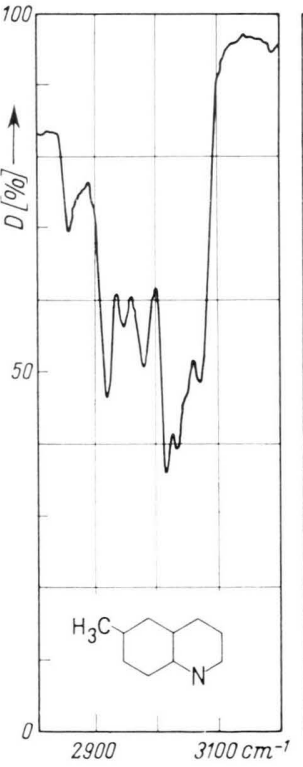

Abb. 7.

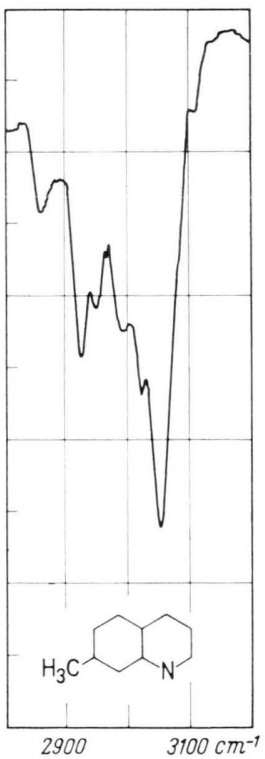

Abb. 8.

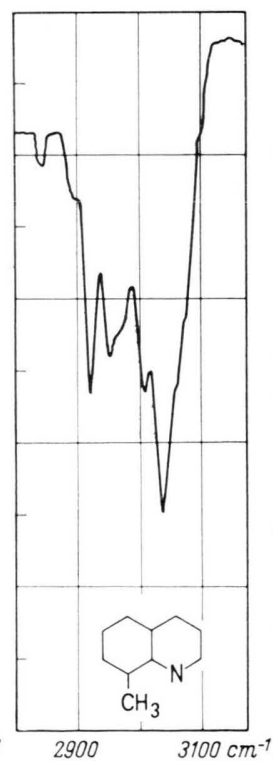

Abb. 3. Banden der CH-Valenzschwingungen von 4-Methylpyridin, $c=0,17$-molar.

Abb. 4. Banden der CH-Valenzschwingungen von 2-Methylchinolin, $c=0,14$-molar.

Abb. 5. Banden der CH-Valenzschwingungen von 4-Methylchinolin, $c=0,14$-molar.

absorbieren, wie auch aus Tab. 2 hervorgeht. Bei folgenden Verbindungen treten in den angegebenen Bereichen keine Banden auf:

Bereich $3082 \pm 12 \mathrm{~cm}^{-1}$ : 2-Methylchinolin, 3-Methylisochinolin, Methylpyrazin; Bereich $3062 \pm$ $12 \mathrm{~cm}^{-1}$ : 4-Methylpyridin, 4-Methylchinolin; Bereich $3034 \pm 8 \mathrm{~cm}^{-1}$ : 2-Methylpyridin, 2-Methylchinolin, 2-Methylchinoxalin, 1-Methyl-, 3-Methylisochinolin; Bereich $3013 \pm 7 \mathrm{~cm}^{-1}$ : 3-Methyl, 4-Methylpyridin, 7-Methylchinolin, 5-Methyl-, 6-Methylchinoxalin. Es dürfte auch nicht ganz ausgeschlossen sein, daß die $v$ - $\mathrm{CH}_{\mathrm{al}}$ des 3- und 4-Methylpyridins bei 2988 und $2993 \mathrm{~cm}^{-1}$ bereits $v-\mathrm{CH}_{\mathrm{ar}} \mathrm{zu}$ zuordnen sind.

Zusätzliche Banden bzw. Schultern um $3105 \mathrm{~cm}^{-1}$ werden beobachtet beim 7-Methyl-, 8-Methylchinolin, 5-Methylchinoxalin und 4-Methylpyridin.

\section{Diskussion}

In reaktiven Methylgruppen sind die H-Atome weniger fest gebunden als in nicht reaktiven Methylgruppen. Diese geringere Bindungsfestigkeit sollte sich auch auf die Kraftkonstante der $v$ - $\mathrm{CH}_{\mathrm{al}}$ auswir- 
Abb. 9.

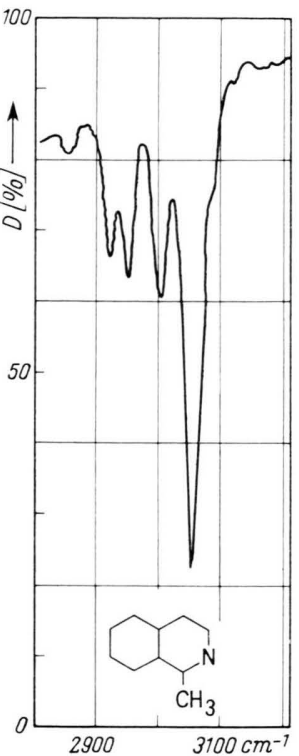

Abb. 11 .

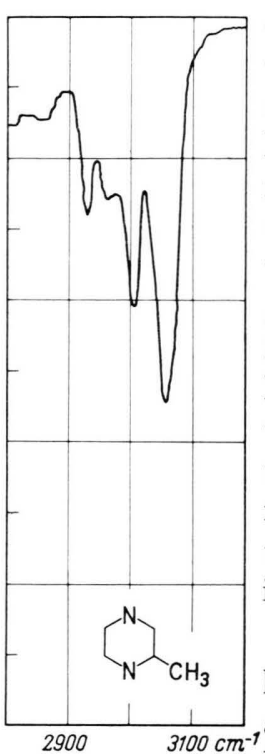

Abb. 9. Banden der CH-Valenzschwingungen von 1-Methylisochinolin, $c=0,12$-molar.

Abb. 10. Banden der CH-Valenzschwingungen von 3-Methylisochinolin, $c=0,12$-molar.

Abb. 11. Banden der CH-Valenzschwingungen von Methylpyrazin, $c=0,13$-molar.

Abb. 12.

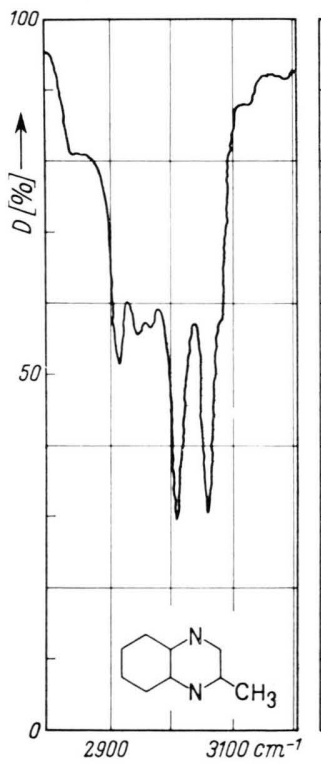

Abb. 13.

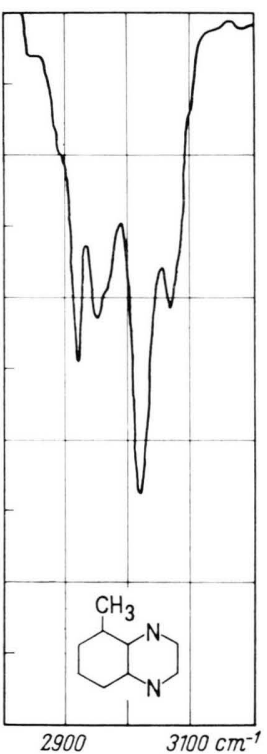

Abb. 14.

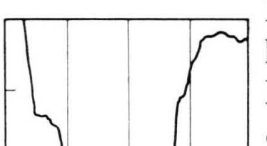

ken. Eine kleinere Kraftkonstante ihrerseits bedingt eine langwellige Verschiebung der Banden der $v-\mathrm{CH}_{\mathrm{al}}$. Untersucht man daraufhin die Lage der Absorptionsmaxima, so läßt sich eine Rotverschiebung bei den Verbindungen mit reaktiver Methylgruppe nicht erkennen. Als Beispiel sei die Bande 2924 士 $6 \mathrm{~cm}^{-1}$ der drei Methylpyridine gewählt: 2-Methylund 4-Methylpyridin haben eine reaktive Methylgruppe. Eine Bande der $v-\mathrm{CH}_{\mathrm{al}}$ liegt bei 2925 bzw. $2924 \mathrm{~cm}^{-1}$. 3-Methylpyridin hat eine nicht reaktive Methylgruppe. Die entsprechende Bande der $v$ - $\mathrm{CH}_{\mathrm{al}}$ wird bei $2924 \mathrm{~cm}^{-1}$ beobachtet. Ein ähnliches Resultat ergibt sich beim Vergleich der Bandenlage der reaktiven und nicht reaktiven Methylderivate der übrigen $N$-Heterocyclen, wie aus Tab. 1 zu ersehen ist.

Neben der Verringerung der Bindungsfestigkeit kann die gesteigerte Reaktivität der Methylgruppe auch auf eine Änderung der Polarität in den CHBindungen zurückgeführt werden. Diese Änderung sollte sich auf die integrale Absorptionsintensität auswirken. Da die integrale Absorptionsintensität der Gesamtbande sicherlich der Summe der maximalen Extinktionskoeffizienten der Teilbanden proportional ist, sollten auch Unterschiede in den Extinktionskoeffizienten der Teilbanden zu erkennen sein. Die angegebenen Extinktionskoeffizienten sind zwar keine absoluten Intensitätsmaße, da aber bei allen Messungen die Aufnahmebedingungen gleich waren, dürften für einen Vergleich untereinander keine Bedenken bestehen. Wie die Tab. 1 erkennen läßt, zeigen sich bei den Extinktionskoeffizienten in der Tat größere Unterschiede als bei der Lage der Maxima der Teilbanden. So ist z. B. bei den Banden der $\nu-\mathrm{CH}_{\mathrm{al}}$ in den Bereichen $2956 \pm 10$ und $2924 \pm 6$ eine Abhängigkeit der Extinktionskoeffizienten von der Stellung der Methylgruppe am $N$-heterocyclischen Ringsystem zu erkennen. Die Banden der reaktionsfähigsten Methylgruppen, z. B. die des Methylpyrazins und 2-Methylchinoxalins, weisen in diesen Bereichen die kleinsten Extinktionskoeffizienten auf. Eine Ausnahme im Bereich $2956 \pm 10 \mathrm{~cm}^{-1}$ macht nur das 4-Methylchinolin, das sich jedoch in dem zweiten Bereich dieser Abstufung relativ gut einfügt.

Dieser Zusammenhang zwischen Reaktivität und Extinktionskoeffizient wird deutlich, wenn man die Summe der Extinktionskoeffizienten der Teilbanden der $v$ - $\mathrm{CH}_{\mathrm{al}}$ bildet, s. Tab. 1 .

Abb. 12. Banden der CH-Valenzschwingungen von 2-Methylchinoxalin, $c=0,23$-molar.

Abb. 13. Banden der CH-Valenzschwingungen von 5-Methylchinoxalin, $c=0,16$-molar.

Abb. 14. Banden der CH-Valenzschwingungen von 6-Methylchinoxalin, $c=0,12$-molar. 


\begin{tabular}{|c|c|c|c|c|c|c|c|c|c|c|c|}
\hline \multirow{2}{*}{ Verbindung } & \multicolumn{11}{|c|}{ Lage der Absorptionsmaxima in $\mathrm{cm}^{-1}$ und molare dekadische Extinktionskoeffizienten } \\
\hline & {$\left[\mathrm{cm}^{-1}\right]$} & $\varepsilon$ & {$\left[\mathrm{cm}^{-1}\right]$} & $\varepsilon$ & {$\left[\mathrm{cm}^{-1}\right]$} & $\varepsilon$ & {$\left[\mathrm{cm}^{-1}\right]$} & $\varepsilon$ & {$\left[\mathrm{cm}^{-1}\right]$} & $\varepsilon$ & $\sum \varepsilon$ \\
\hline 2-Methylpyridin & & & 2962 & 12,4 & 2925 & 16,1 & 2870 & 5,4 & & & 34 \\
\hline 3-Methylpyridin & $2988 ?$ & 22,1 & 2945 & 14,2 & 2924 & 18,3 & 2860 & 7,5 & & & 58 \\
\hline 4-Methylpyridin & $2993 ?$ & 22,7 & 2966 & 12.5 & 2924 & 14,7 & 2873 & $4,0 \mathrm{sh}$ & 2895 & $5,0 \mathrm{sh}$ & 59 \\
\hline 2-Methylchinolin & & & 2952 & 11,9 & 2923 & 15,8 & 2860 & $2,9 \mathrm{sh}$ & & & 31 \\
\hline 4-Methylchinolin & 2987 & 20.4 & 2958 & 18.6 & 2929 & 13.4 & 2860 & 3.3 & 2910 & $7.0 \mathrm{sh}$ & 63 \\
\hline 6-Methylchinolin & 2983 & 20,5 & 2950 & 17.6 & 2924 & 23,8 & 2860 & 11,7 & & & 74 \\
\hline inolin & 2997 & 18,2 & 2955 & 16,5 & 2929 & 20,7 & 2866 & 10,6 & & & 66 \\
\hline 8-Methylchinolin & 2980 & $20,4 \mathrm{sh}$ & 2959 & 22,3 & 2927 & 26,8 & 2847 & 9,1 & 2900 & 10,5 & 89 \\
\hline 1-Methylisochinolin & & & 2954 & 16,6 & 2923 & 14,6 & 2861 & 7,4 & & & 39 \\
\hline 3-Methylisochinolin & 2986 & 29,8 & 2957 & 19,3 & 2923 & 23,4 & 2875 & 9,0 & 2900 & $10,9 \mathrm{sh}$ & 93 \\
\hline Methylpyrazin & & & 2963 & 9,6 & 2928 & 11,1 & 2855 & 4,8 & & & 26 \\
\hline 2-Methylchinoxalin & 2979 & 10,6 & 2955 & 10,9 & 2923 & 12,3 & 2855 & $3.8 \mathrm{sh}$ & & & 38 \\
\hline 5-Methylchinoxalin & & & 2959 & 13,7 & 2923 & 19,5 & 2850 & 1,2 & 2900 & $6,1 \mathrm{sh}$ & 41 \\
\hline 6-Methylchinoxalin & 2977 & 15.8 & 2946 & 12.8 & 2918 & 16.7 & 2880 & 5,3 & & & 51 \\
\hline 1-Methyl & 2972 & 21,6 & 2947 & 23,6 & 2932 & $20,6 \mathrm{sh}$ & 2861 & 12,7 & 2910 & 16,8 & 95 \\
\hline 2-Methylnaphthalin & 2973 & 20,5 & 2946 & 19,3 & 2923 & 25,6 & 2861 & 13,2 & & & 79 \\
\hline
\end{tabular}

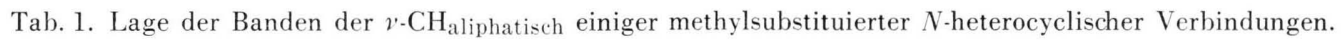

\begin{tabular}{|c|c|c|c|c|c|c|c|c|c|c|}
\hline \multirow{2}{*}{ Verbindung } & \multicolumn{10}{|c|}{ Lage der Absorptionsmaxima in $\mathrm{cm}^{-1}$ und molare dekadische Extinktionskoeffizienten } \\
\hline & {$\left[\mathrm{cm}^{-1}\right]$} & $\varepsilon$ & {$\left[\mathrm{cm}^{-1}\right]$} & $\varepsilon$ & {$\left[\mathrm{cm}^{-1}\right]$} & $\varepsilon$ & {$\left[\mathrm{cm}^{-1}\right]$} & $\varepsilon$ & {$\left[\mathrm{cm}^{-1}\right]$} & $\varepsilon$ \\
\hline 2-Methylpyridin & 3083 & 17,9 & 3068 & 19,4 & & & 301 & 27,9 & & \\
\hline 3-Methylpvridin & 3092 & 11,0 & 3056 & $17,5 \mathrm{sh}$ & 3031 & 31.1 & $2988 ?$ & 22,1 & & \\
\hline 4-Methylpyridin & 3071 & 26,4 & & & 3030 & 22,2 & $2993 ?$ & 22,7 & 3100 & $6,2 \mathrm{sh}$ \\
\hline 2-Methylchinolin & & & 3057 & 34,2 & & & 3011 & 12,8 & & \\
\hline 4-Methylchinolin & 3071 & 26,9 & & & 3042 & 28.9 & 3010 & 15,8 & & \\
\hline 6 -Methylchinolin & 3086 & 22,0 & 3050 & $23,6 \mathrm{sh}$ & 3038 & 29,4 & 3020 & 31 , & & \\
\hline 7-Methylchinolin & 3082 & $15,1 \mathrm{sh}$ & 3062 & 39,1 & 3028 & 23,7 & & & 3107 & 4,9 \\
\hline 8-Methylchinolin & 3081 & $19,8 \mathrm{sh}$ & 3063 & $26,2 \mathrm{sh}$ & 3044 & 41,9 & 3015 & 26,3 & 3104 & $6,7 \mathrm{sh}$ \\
\hline 1-Methylisochinolin & 3094 & $10,2 \mathrm{sh}$ & 3056 & $5.5,4$ & & & 3008 & 17,9 & & \\
\hline 3-Methylisoc & & & 3058 & 38,1 & & & 3010 & $15,2 \mathrm{sh}$ & & \\
\hline Methylpy & & & 30.58 & 25,8 & 3039 & $14,8 \mathrm{sh}$ & 3006 & 17,1 & & \\
\hline 2-Methylchino & 3087 & $10,5 \mathrm{sh}$ & 3068 & 22,4 & & & 3019 & 22,8 & & \\
\hline 5-Methylchin & 3093 & $11,7 \mathrm{sh}$ & 3073 & 14,8 & 3026 & 31,0 & & & 3110 & $4,2 \mathrm{sh}$ \\
\hline 6-Methylchinoxalin & 3084 & $4,3 \mathrm{sh}$ & 3068 & $15,2 \mathrm{sh}$ & 3036 & 26,6 & & & & \\
\hline 1-Methylnaphthalin & 3070 & 42,7 & 3053 & 39,3 & & & 3011 & 17,4 & 3101 & 10,6 \\
\hline 2-Methylnaphthalin & 3090 & $11.9 \mathrm{sh}$ & 3057 & 57,1 & 3023 & 27,4 & & & & \\
\hline
\end{tabular}

Tab. 2. Lage der Banden der $v$ - $\mathrm{CH}_{\mathrm{ar}}$ einiger methylsubstituierter $N$-heterocyclischer Verbindungen.

Bei Verbindungen mit einer reaktiven Methylgruppe, z. B. Methylpyrazin $\left(\sum \varepsilon_{\max }=26\right)$ ist die Summe der Extinktionskoeffizienten im allgemeinen kleiner als bei den Verbindungen mit nicht reaktiven Methylgruppen, z. B. 7-Methoxylchinolin $\left(\sum \varepsilon_{\max }\right.$ $=66)$. In dieses Schema lassen sich nicht einordnen: 4-Methylpyridin $\left(\sum \varepsilon_{\max }=59\right)$, 4-Methylchinolin $\left(\Sigma \varepsilon_{\max }=63\right)$ und 3-Methylisochinolin $\left(\sum \varepsilon_{\max }=\right.$ 93), deren $\sum \varepsilon_{\max }$ weit höher liegen als die der anderen Verbindungen mit reaktiver Methylgruppe. Es ist aber bekannt, daß 4-Methylpyridin, 4-Methylchinolin und 3-Methylisochinolin nicht so reaktionsfähig sind wie die entsprechenden 2-Methyl-Derivate bzw. das 1-Methyl-Derivat. Von den Verbindungen mit nichtreaktiver Methylgruppe fügen sich 5-Methylchinoxalin $\left(\sum \varepsilon_{\max }=41\right)$ und 6-Methylchinoxalin $\left(\sum \varepsilon_{\max }=51\right)$ nicht so gut in das Schema. Berücksichtigt man, daß beim 4-Methylpyridin die $v-\mathrm{CH}_{\mathrm{a} l}$ bei $2993 \mathrm{~cm}^{-1}$ evtl. einer $v-\mathrm{CH}_{\mathrm{ar}}$ entspricht, so würde sich $\Sigma \varepsilon$ erniedrigen und damit mit dem Wert für 2-Methylpyridin übereinstimmen.

Für das Interesse an dieser Arbeit danken wir dem Institutsdirektor, Herrn Prof. Dr. Dr. h. c. R. SuHRmans, sehr herzlich. Der Deuts chen Forschungsgemeinschaft danken wir für die apparative Unterstützung sowie dem Verband der Chemi schen Industrie für die Gewährung eines Liebig-Stipendiums. 